\title{
Finite Element Simulation of a Taylor Bubble in Two-Phase Gas-Liquid Slug Flows using Petrov- Galerkin Formulation
}

\author{
H. A. Abubakar ${ }^{1 *}$, A. Yusuf ${ }^{2}$, Y. Sanusi ${ }^{3}$, H. A. Dandajeh ${ }^{3}$ \\ ${ }^{1}$ Department of Chemical Engineering, Ahmadu Bello University, Zaria, Nigeria. \\ ${ }^{2}$ Department of Mathematics, Federal University of Technology, Minna, Nigeria. \\ ${ }^{3}$ Department of Mechanical Engineering, Ahmadu Bello University, Zaria, Nigeria.
}

ABSTRACT: Petrov-Galerkin finite element scheme for systematic analysis of the dynamics of a rising Taylor bubble and general free surface flow problems is derived and implemented. The validity of the scheme is confirmed by simulating the buoyancy-driven motion of a Taylor bubble through a stagnant Newtonian liquid in a vertical pipe characterised by dimensionless inverse viscosity number and Eötvös number of magnitude 111 and 189, respectively. Comparison of the numerical results for the steady state features defining the nose, film, and bottom regions around the bubble with the experiment shows a good agreement between the numerical simulation and the experiment. The percentage deviation of the numerical computed rise velocity, equilibrium film thickness, and stabilisation length ahead of the bubble from the experimental determined values are $8.4 \%, 2.3 \%$, and $9.5 \%$, respectively.

KEYWORDS: Taylor bubble, finite element, slug flow, CFD, Petrov-Galerkin

\section{INTRODUCTION}

Two-phase gas-liquid flows refer to the interactive flow of gas and liquid, as a result of which, the interface between the two phases is influenced by their motion. They are considered as the most complex of the four types of two-phase flows because of the deformable nature of the interface and the compressible nature of the gas phase. Different classes of interface distribution, termed flow regimes or patterns, can be defined in two-phase gas-liquid flows. In the vertical flow of gas and liquid through pipes, increasing the gas-phase flow rate at a constant liquid phase flow rate results in the following flow regimes: bubbly flow, slug or plug flow, churn flow and annular flow (Figure 1).

Among the different flow patterns, slug flow has attracted a lot of attention in literature. It features periodic-like flow of large bullet-shaped elongated bubbles separated by regions containing liquid, which may have dispersed smaller bubbles. These large elongated bubbles are known as Taylor bubbles and have found applications in numerous processes such as the production and transportation of hydrocarbons, the emergency cooling of nuclear reactors, the flow of blood in blood vessels, the cooling of electronics, the eruption of volcano and the boiling and condensing process in power plants (Taha \& Cui, 2006; Magnini et al., 2013; Capponi et al., 2016; Pering \& McGonigle, 2018). The near-periodicity nature of the gasliquid slug flow in vertical pipes has made the investigation of *Corresponding author: haabubakar@abu.edu.ng the behaviour of a single Taylor bubble a paradigm for understanding of the flow regime (Pringle et al., 2015).

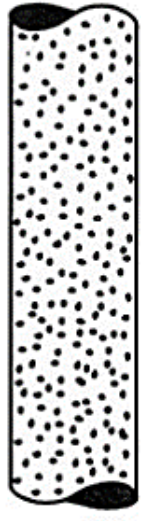

Bubbly

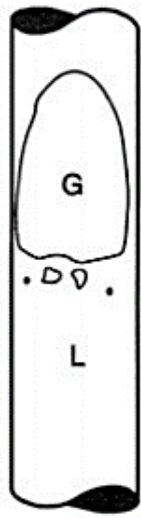

Slug

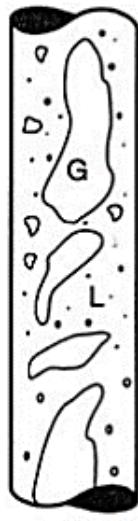

Churn

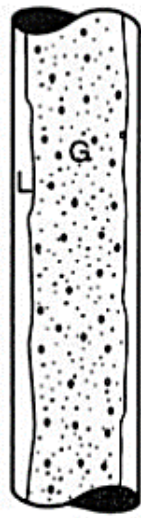

Annular
Figure 1: Flow patterns of gas-liquid flow in vertical pipes. Reproduced from Fazel (2017).

The morphology of a rising Taylor bubble is axisymmetric and can be divided into three different regions, namely nose, film, and bottom, with each region having definite features that characterise them. The nose region is nearly hemispherical while the body region is cylindrical surrounded by a falling

doi: http://dx.doi.org/10.4314/njtd.v18i3.7 
liquid film, which is either developing or fully developed. The bottom region comprises a tail subregion, which may be convex, flat, or concave, and may have a wake subregion, which could be laminar, transitional, or turbulent.

Several research works have been done to understand the hydrodynamics of Taylor bubble as it pertains to its different regions because of the numerous applications of the slug flow. While extensive experimental and a number of theoretical studies (Bugg \& Saad, 2002; Funada et al., 2005; Nogueira et al., 2006b; Llewellin et al., 2012; Fabre, 2016;Abubakar \& Matar, 2021a, 2021b) have been carried out on the features that defined the aforementioned morphological regions, there are still much more to be understood, particularly in the area of systematically studying the influence of the dimensionless parameters that characterised the flow on the dynamics of the bubble and in the area of providing deep insight into the flow field around and within the bubble. In these areas, numerical simulations have proven to be an efficient and cost-effective means, and in recent years, there have been a number of computational studies that have attempted to address them (Bugg \& Saad, 2002; Taha \& Cui, 2006; Lu \& Prosperetti, 2009; Kang et al., 2010; Anjos et al., 2014; Lizarraga-Garcia et al., 2017).

Complex numerical simulations of two-phase flows, or multiphase flows in general, are largely carried out based on either finite element or finite volume discretisation, with a large number of studies using the finite volume technique because of its simplicity. However, the advancement in mesh generation and adaptivity techniques, which allows refinement in regions that matters, makes the finite element approach a great choice, despite its rigorous mathematics. A number of numerical simulations of the dynamics of a large elongated bubble in liquid have been carried out based on finite element discretisation, particularly using Galerkin method (Feng, 2008, 2009; Anjos et al., 2014). Studies have shown that Galerkin method of solving Navier-Stokes equations is only effective for low values of Reynolds number (Reddy \& Gartling, 2010). For convection-dominated problems, the solution exhibits oscillations in the velocity field leading to non-physical results and eventually prevents the solution from converging. For a general problem, be it viscous, convection or surface tension dominated, streamline-upwind/Petrov-Galerkin (SUPG) stabilization technique is one of the recommended approaches to avoid the challenges associated with convection-dominated problems (Reddy \& Gartling, 2010; Pepper \& Heinrich, 2017). In view of this, Petrov-Galerkin finite element scheme is developed and implemented within the open source finite element package FreeFem++ (Hecht, 2012) for a rising Taylor bubble in stagnant Newtonian liquids.

\section{FINITE ELEMENT MODEL OF A RISING TAYLOR BUBBLE}

\section{A. Problem Description}

An axisymmetric Taylor bubble of constant volume $V_{b}$ rising at a constant velocity of magnitude $U_{b}$ in a liquid of constant density $\rho$, viscosity $\mu$, and interfacial tension $\gamma$ in a vertical pipe of radius $\mathrm{R}$ is considered. It is assumed that the density, $\rho_{\mathrm{g}}$, and viscosity, $\mu_{\mathrm{g}}$, of the gas phase (bubble) are very small compared to those of the liquid. Therefore, the dynamics in the gas phase is approximated to a constant pressure $\mathrm{P}_{\mathrm{b}}$ and the influence of the gas phase is therefore restricted to the interface between the liquid and the gas phase $\Gamma_{\mathrm{b}}$. As a result, only the flow fields in the liquid phase and the bubble pressure are determined. This concept of constant bubble pressure in the gas phase is relatively common in the literature (Tsamopoulos et al., 2008; Fraggedakis et al., 2016) and has been shown to reasonably describe the dynamics of Taylor bubbles (Feng, 2008; Lu \& Prosperetti, 2009; Kang et al., 2010). Cylindrical coordinates system is adopted, so that the coordinates along and perpendicular to the axis of symmetry are $\mathrm{z}$ and $\mathrm{r}$, respectively, with the origin of $\mathrm{z}$ chosen as the nose of the bubble. The problem therefore is to find the rise velocity $\left(\mathrm{U}_{\mathrm{b}}\right)$ pressure $\left(\mathrm{P}_{\mathrm{b}}\right)$, and shape of a Taylor bubble as it rises through a stagnant liquid of density $(\rho)$, viscosity $(\mu)$, and interface tension $(\gamma)$ in a pipe of diameter $\mathrm{D}=2 \mathrm{R}$ so that its volume $\left(\mathrm{V}_{\mathrm{b}}\right)$ remains constant.

\section{B. Governing Equations and Boundary Conditions}

For an isothermal flow, the motion of an incompressible Newtonian liquid in a domain $\Omega$ with boundary $\Gamma$ at steady state is governed by the time-independent Navier-Stokes and continuity equations, supplemented with appropriate boundary conditions. By making the length, velocity, and pressure variables in these equations dimensionless with the characteristic length, velocity, and pressure scales, taken to be $\mathrm{D}, \sqrt{g D}$ and $\rho \mathrm{gD}$, respectively, the dimensionless governing equations together with the boundary conditions in a frame translated with the velocity $\left(\mathbf{u}_{\mathrm{b}}\right)$ of the bubble nose become

\section{Momentum Equation}
$(\mathbf{u} . \boldsymbol{\nabla}) \mathbf{u}-\boldsymbol{\nabla} . \mathbf{T}=\mathbf{0}$
in $\Omega$

\section{Continuity Equation}
$\boldsymbol{\nabla} \cdot \mathbf{u}=0$
in $\Omega$

where $\Omega$ denotes the domain of interest, $\mathbf{u}$ is the vector of the fluid velocity in the moving frame of reference, $\mathbf{T}$ is the stress tensor, and $\nabla$ is the gradient operator in the cylindrical coordinate system. The constitutive equations that relate the stress tensor to the dependent variables of the system of equations are

$$
\begin{aligned}
& \mathbf{T}(\mathbf{u}, \mathrm{p})=-\mathrm{pI}+2 \mathrm{Nf}^{-1} \mathbf{E}(\mathbf{u}) \\
& \mathbf{E}(\mathbf{u})=1 / 2\left[\nabla(\mathbf{u})+\nabla(\mathbf{u})^{\mathrm{T}}\right]
\end{aligned}
$$

where p represents the dynamic pressure, $\mathbf{I}$ is the unit tensor, $\mathrm{Nf}$ is the dimensionless inverse viscosity number defined in (4), $\mathbf{E}$ is the rate of deformation tensor, and (. $)^{\mathrm{T}}$ is the transpose operator. 


$$
\begin{aligned}
& N f=\frac{\rho\left(g D^{3}\right)^{1 / 2}}{\mu} \\
& E o=\frac{\rho g D^{2}}{\gamma}
\end{aligned}
$$

Appropriate boundary conditions are prescribed at the boundary of the domain to make the problem well posed and the solution unique. The boundary denoted by $\Gamma$ can be divided into $\Gamma_{\text {in }}, \Gamma_{\text {out }}, \Gamma_{\text {wall }}, \Gamma_{\text {sym }}$ and $\Gamma_{\mathrm{b}}$ as shown in Figure 2. The subscripts in, out, wall, sym and $b$ stand for the inlet, outlet, wall, symmetry, and bubble boundaries, respectively. At the wall, no-slip and no-penetration boundary conditions are imposed, while at the inlet, prescribed values are specified for the velocity components, which for the stagnant liquid are zeros. Boundary conditions of the types at the wall and inlet where the values of the primary variables are specified are called Dirichlet, or essential boundary conditions. At the outlet of the domain, a zero traction condition, also known as outlet condition, is imposed.

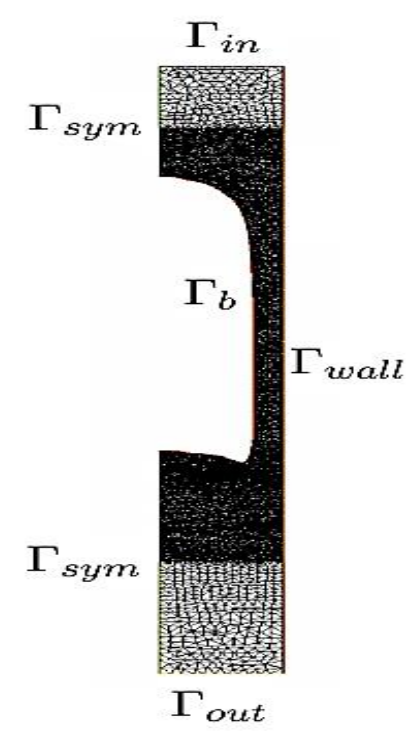

Figure 2: Discretised simulation domain showing the boundaries.

The conditions prescribed at the symmetry axis are zero normal velocity and shear stress. Finally, at the gas-liquid interface, normal stress, tangential stress, and kinematic boundary conditions are imposed. In (10a), $\kappa$ is the curvature of the interface, $\mathrm{z}$ is the axial component of the spatial coordinates of the interface, and Eo is the dimensionless Eötvös number defined in (5). It should be noted that gravity appears in (10a) as $\mathrm{z}$ because the hydrostatic component of the pressure has been subtracted from the total pressure, leaving only the hydrodynamic part. In addition, it is noteworthy that $\mathbf{r}_{\mathrm{b}}$ in (10c) represents the position vector of all points on the gas-liquid interface and $\mathbf{n}$ in (8) - (10) is the unit normal vector for the corresponding boundaries.

\section{Boundary conditions}

Wall: $\mathbf{u}=-\mathbf{u}_{\mathrm{b}} \quad$ on $\quad \Gamma_{\text {wall }}$

Inlet:

$\mathbf{u}=-\mathbf{u}_{\mathrm{b}} \quad$ on $\quad \Gamma_{\text {in }}$

Outlet:

n. $\mathbf{T}=\mathbf{0} \quad$ on $\quad \Gamma_{\text {out }}$

Symmetry axis:
u. $\mathbf{n}=0$
on
$\Gamma_{\text {sym }}$
n. $\mathbf{T} \times \mathbf{n}=\mathbf{0}$
on $\quad \Gamma_{\text {sym }}$

Gas-Liquid Interface:

$\begin{array}{lcc}\mathbf{n} . \mathbf{T} \cdot \mathbf{n}+\mathrm{P}_{\mathrm{b}}-\mathrm{z}-\mathrm{Eo}^{-1} \mathrm{k}=0 & \text { on } & \Gamma_{b} \\ \mathbf{n} . \mathbf{T} \times \mathbf{n}=0 & \text { on } & \Gamma_{b} \\ \frac{\mathrm{d} \mathbf{r}_{\mathrm{b}}}{\mathrm{dt}} \cdot \mathbf{n}-\mathbf{u} \cdot \mathbf{n}=0 & \text { on } & \Gamma_{b}\end{array}$

A close look at the system of Eq. (1)-(10) shows that the system is similar to what would be obtained for free surface flow; the only difference is that bubble pressure $\left(\mathrm{P}_{\mathrm{b}}\right)$, in equation(10a) replaces the ambient pressure, $P_{a}$, for free surface flow. Hence, the scheme that is described in Sections II.C and II.D can be adapted to solve free surface flow problems. For a consistent system, an additional equation is needed to determine the unknown dimensionless bubble pressure. The equation is the constraint that the dimensionless bubble volume, $\mathrm{V}_{\mathrm{b}}$, remains constant. Applying Gauss' theorem to the divergence of the position vector,

$\mathrm{V}_{\mathrm{b}}+\frac{2 \pi}{3} \oint_{\Gamma_{\mathrm{b}}}\left[\mathbf{r}_{\mathrm{b}} \cdot \mathbf{n}\right] \mathrm{d} \Gamma_{\mathrm{b}}=0$

\section{Weak Formulations}

Eq. (1) - (11) are the continuous form of the mathematical model describing the problem. Solving equations (1) - (2) under the boundary conditions (6) - (11) using the finite element method requires that the equations be transformed into their weak forms, the dependent variables in the equations approximated using suitable basis functions and the domain divided into subdomains around which the approximated variables are defined to give a set of algebraic relations among the unknown parameters of the approximations, known as finite element models of the equations. The transformation is carried out by multiplying the governing equations for each variable with their corresponding test functions and integrating over the domain, reducing the order of integration by integrating by part, and finally, incorporating the boundary conditions into the resulting relations. 
Let $\Phi \in \mathrm{H}^{1}{ }_{0}$ and $\varphi \in \mathrm{L}^{2}{ }_{0}$ be the test functions corresponding to $\mathbf{u} \in \mathrm{H}^{1}$ and $\mathrm{p} \in \mathrm{L}^{2}$, respectively. $\mathrm{H}^{1}$ and $\mathrm{L}^{2}$ are Sobolev and Lebesgue spaces while $\mathrm{H}_{0}^{1}$ and $\mathrm{L}_{0}^{2}$ are the corresponding subspaces as defined in Abubakar (2019). The weighted residual forms of the governing equations are obtained by taking the inner product of equation (1) with $\boldsymbol{\Phi}$, multiplying Eq. (2) with $\varphi$ and integrating the equations over the domain.

$$
\begin{aligned}
& \int_{\Omega}\{[(\mathbf{u} . \boldsymbol{\nabla}) \mathbf{u}] . \Phi-[\boldsymbol{\nabla} . \mathbf{T}] . \Phi\} \mathrm{d} \Omega=0 \\
& \int_{\Omega}\{(\boldsymbol{\nabla} \cdot \mathbf{u}) \varphi\} \mathrm{d} \Omega=0
\end{aligned}
$$

Integrating by part the last term on the left side of (12), enforcing the boundary conditions (6)-(9), taking into consideration that $\boldsymbol{\Phi} \in \mathrm{H}^{1}{ }_{0}$, which makes $\boldsymbol{\Phi}$ to be zero on the boundaries $\Gamma_{\text {in }}$ and $\Gamma_{\text {wall }},(12)$ gives

$$
\begin{aligned}
\int_{\Omega}\left\{[(\mathbf{u} . \boldsymbol{\nabla}) \mathbf{u}] . \boldsymbol{\Phi}+2 \mathbf{N f}^{-1} \mathbf{E}(\mathbf{u}): \mathbf{E}(\boldsymbol{\Phi})\right\} \mathrm{d} \Omega \\
\quad-\int_{\Omega}\{\mathrm{p}(\boldsymbol{\nabla} . \boldsymbol{\Phi})\} \mathrm{d} \Omega-\int_{\Gamma_{\mathrm{b}}}\{\mathbf{n} . \mathbf{T} . \boldsymbol{\Phi}\} \mathrm{d} \Gamma_{\mathrm{b}}=0
\end{aligned}
$$

Boundary conditions (10a) and (10b) are imposed by decomposing the traction term, the last term on the left hand side, in (14) into its normal and tangential components (Pozrikidis, 2011) to give

$$
\begin{aligned}
& \int_{\Omega}\left\{[(\mathbf{u} . \boldsymbol{\nabla}) \mathbf{u}] . \Phi+2 \mathrm{Nf}^{-1} \mathbf{E}(\mathbf{u}): \mathbf{E}(\boldsymbol{\Phi})\right\} \mathrm{d} \Omega- \\
& \int_{\Omega}\{\mathrm{p}(\boldsymbol{\nabla} \cdot \boldsymbol{\Phi})\} \mathrm{d} \Omega-\int_{\Gamma_{\mathrm{b}}}\left\{\left[\mathrm{Eo}^{-1} \kappa+\mathrm{z}\right] \mathbf{n} . \boldsymbol{\Phi}\right\} \mathrm{d} \Omega+ \\
& \int_{\Gamma_{\mathrm{b}}}\left\{\mathrm{P}_{\mathrm{b}} \mathbf{n} . \boldsymbol{\Phi}\right\} \mathrm{d} \Gamma_{\mathrm{b}}=0
\end{aligned}
$$

Eqs. (13) and (14) are the weak forms of the continuity and the momentum equations with the boundary conditions incorporated except (10c) and (11). For the stability of the solution, the surface tension effect on the interface must be treated implicitly and (10c) is the additional equation that determines the interface deformation. A pseudo-time stepping technique is used and the deformation is taken to be in the direction that is normal to the interface so that (10c) is written as

$$
\mathbf{x}=\delta \mathbf{r}_{\mathrm{b}}=\mathbf{r}_{\mathrm{b}}^{\mathrm{n}}-\mathbf{r}_{\mathrm{b}}^{\mathrm{n}-1}=\delta \mathrm{t}(\mathbf{u} \cdot \mathbf{n}) \mathbf{n}
$$

and the weak form becomes

$$
\int_{\Gamma_{\mathrm{b}}}\{\mathbf{x} \cdot \boldsymbol{\Psi}-\delta \mathrm{t}(\mathbf{u} \cdot \mathbf{n}) \mathbf{n} \cdot \boldsymbol{\Psi}\} \mathrm{d} \Gamma_{\mathrm{b}}=0
$$

where $\boldsymbol{\Psi}$ is the test function for the interface deformation vector. Lastly, (16) is substituted in (11) to arrive at

$$
\mathrm{V}_{\mathrm{b}}-\mathrm{V}_{\mathrm{b}}^{\mathrm{n}-1}+\frac{2 \pi}{3} \oint_{\Gamma_{\mathrm{b}}}[\mathbf{x} \cdot \mathbf{n}] \mathrm{d} \Gamma_{\mathrm{b}}=0
$$

Eqs. (13), (15), (17) and (18) form the consistent weak formulation for the mathematical models of the rising Taylor bubble with negligible dynamics in the gas-phase that allows the determination of the pressure field, velocity field, interface deformation and the bubble pressure, respectively. It is noted that the aforementioned equations are the same for Galerkin finite element formulation (Abubakar, 2019). However, unlike in Galerkin formulation, the test functions for the velocity and interface deformation in Petrov-Galerkin formulation are given as

$$
\begin{aligned}
& \boldsymbol{\Phi}=\boldsymbol{\Phi}^{*}+\frac{\mathrm{h}}{\left|\mathbf{u}^{0}\right|} \mathbf{u}^{0} \cdot \boldsymbol{\nabla} \boldsymbol{\Phi}^{*} \\
& \boldsymbol{\Psi}=\boldsymbol{\Psi}^{*}+\frac{\mathrm{h}}{\left|\mathbf{u}^{0}\right|} \mathbf{u}^{0} \cdot \boldsymbol{\nabla} \boldsymbol{\Psi}^{*}
\end{aligned}
$$

where $\boldsymbol{\Phi}^{*}$ and $\boldsymbol{\Psi}^{*}$ are the standard Galerkin formulation test functions, $\mathrm{h}$ is the mesh length, and $\mathbf{u}^{0}$ is the streamwise vector of velocity. Petrov-Galerkin formulation in which the test functions are of the form (19) is called streamline-upwinded Petrov-Galerkin (SUPG) finite element stabilisation technique.

\section{Discretised Formulations}

The derived weak formulations in Section II.C are discretised using suitable finite elements and the unknowns represented using a stable combination of finite element functions. The domain is triangulated based on DelaunayVoronoi algorithm and the finite element combinations used are Taylor-Hood element for the velocity-pressure coupling, i.e piecewise quadratic continuous element, $\mathrm{P}_{2}$ for the velocity components and a piecewise linear continuous element, $\mathrm{P}_{1}$, for pressure. For interface deformation, following Ban̈sch (2001), a piecewise quadratic continuous element was used. Thus, the final discrete finite element models are

$$
\begin{aligned}
& \left\langle[(\mathbf{u} . \boldsymbol{\nabla}) \mathbf{u}] . \Phi+2 \mathrm{Nf}^{-1} \mathbf{E}(\mathbf{u}): \mathbf{E}(\boldsymbol{\Phi})\right\rangle_{\Omega^{\mathrm{e}}}-\langle\mathrm{p}(\boldsymbol{\nabla} \cdot \boldsymbol{\Phi})\rangle_{\Omega^{\mathrm{e}}}- \\
& \mathrm{Eo}^{-1}\langle\mathrm{kn} \cdot \boldsymbol{\Phi}\rangle_{\Gamma_{\mathrm{b}}^{\mathrm{e}}}-\left\langle\mathrm{P}_{\mathrm{b}} \mathbf{n} \cdot \boldsymbol{\Phi}\right\rangle_{\Gamma_{\mathrm{b}}^{\mathrm{e}}}=\langle\mathrm{zn} \cdot \boldsymbol{\Phi}\rangle_{\Gamma_{\mathrm{b}}^{\mathrm{e}}} \\
& \langle(\boldsymbol{\nabla} \cdot \mathbf{u}) \varphi\rangle_{\Omega^{\mathrm{e}}}=0
\end{aligned}
$$

$-\delta \mathrm{t}\langle(\mathbf{u} \cdot \mathbf{n}) \mathbf{n} \cdot \boldsymbol{\Psi}\rangle_{\Gamma_{\mathrm{b}}^{\mathrm{e}}}+\langle\mathbf{x} \cdot \boldsymbol{\Psi}\rangle_{\Gamma_{\mathrm{b}}^{\mathrm{e}}}=0$

$\langle\mathbf{x} \cdot \mathbf{n}\rangle_{\Gamma_{\mathrm{b}}^{\mathrm{e}}}=\frac{-3}{2 \pi}\left(\mathrm{V}_{\mathrm{b}}-\mathrm{V}_{\mathrm{b}}^{\mathrm{n}-1}\right)$

$\mathrm{k}=\mathrm{k}^{0}+\frac{1}{\mathrm{r}^{0}} \frac{\mathrm{d}}{\mathrm{ds} \mathrm{s}^{0}}\left[\mathrm{r}^{0}\left(\mathbf{n}^{0} \cdot \frac{\mathrm{dx}}{\mathrm{ds} \mathrm{s}^{0}}\right)\right]-\left[\mathrm{k}_{1}^{0}\left(\mathrm{t}^{0} \cdot \frac{\mathrm{dx}}{\mathrm{ds^{0 }}}\right)+\mathrm{k}_{2}^{0} \frac{\mathrm{x}_{\mathrm{r}}}{\mathrm{r}^{0}}\right]$

where $\mathbf{n}^{0}$ and $\mathbf{t}^{0}$ are the unit normal and tangent vectors to the currently known interface; $\mathrm{ds}^{0}$ is the elemental arc length of the currently known interface with position vector $\mathbf{r}^{0}$ whose components are $r^{0}$ and $z^{0} ; \kappa^{0}$ is the total curvature of the currently known interface and is related to the two principal curvatures of the interface in $\mathrm{r}-\mathrm{Z}$ and $\mathrm{r}-\theta$ planes, $\kappa^{0}{ }_{1}$ and $\kappa^{0}{ }_{2}$, respectively as in Eq. (25).

$\kappa^{o}=\kappa_{1}^{0}+\kappa_{2}^{0}$ 


\section{FINITE ELEMENT MODEL SIMULATION}

\section{A. Steady State Model Simulations}

The discrete formulation given by the system of equations (20)-(25) is non-linear in velocity and interface position due to the presence of the convective terms and the implicit treatment of curvature, respectively. For this reason, the equations are solved in three stages, using Newton's method.

First, for a given $\mathrm{Nf}$ and Eo, initial guesses for the bubble rise velocity, the flow field variables, and position vector of the interface, denoted as $\mathrm{U}_{\mathrm{b}},\left(\mathbf{u}^{0}, \mathrm{p}^{0}\right)$, and $\mathbf{r}_{\mathrm{b}}^{0}$ are provided. The variables in the equations and the interface curvature are linearised about the given initial guesses to obtain linear discrete equations which are solved iteratively in a frame of reference that moves with velocity $\mathbf{u}_{b}=\mathbf{U}_{b} \mathbf{i}_{\mathbf{z}}$ until the solution converges. The Newton iteration is deemed to have converged when the $\mathrm{L}_{2}$ norm of the difference between the solutions of two successive iterations is less than $10^{-15}$. In the second stage, the rise velocity of the bubble is estimated from the velocity field solution computed in the first stage. The velocity field is converted from a moving frame of reference to a fixed frame (26a) and the value of the axial velocity at the nose of the bubble, which is fixed as the origin, is extracted and set as the updated bubble velocity (26b). This stage is summarised as

$\mathbf{u}^{\text {fixed }}=\mathbf{u}+\mathbf{u}_{\mathbf{b}}=\mathrm{u}_{\mathrm{r}} \mathbf{i}_{\mathrm{r}}+\left(\mathrm{u}_{\mathrm{z}}-\mathrm{U}_{\mathrm{b}}\right) \mathbf{i}_{\mathrm{z}}$

$U_{b}=-u_{z}^{\text {fixed }}(0,0)$

Lastly, the domain is deformed by solving a linear elasticity equation based on the elastic mesh update technique, described in Abubakar (2019), using the computed solution for the interface deformation field in the first stage, as the boundary condition.

The three stages of the solution are continuously repeated using the updated initial guess for the variables, bubble rise velocity and interface position vector until the $\mathrm{L}_{2}$ norm of the normal velocity at the interface is less than $10^{-4}$. In addition, it is expected that the average error for the flow field, rise velocity, and interface position is less than $10^{-6}$. On termination of the iterative process, the flow field solution, rise velocity, and interface position vector for the last iteration are declared as the solutions for the given dimensionless parameters. From the interface position vector, the steady state bubble shape is generated. It is noteworthy that occasionally, there is the need to carry out remeshing which is done by adapting the mesh around the interface to the curvature of the interface and the by adapting the mesh around the interface to the curvature of the interface and the flow field around it, while the other boundaries of the domain are maintained at a fixed number of mesh points that have been previously verified to be sufficient in Abubakar (2019).

\section{B. Steady State Model Validation and Discussions}

The model derived in Section II and the numerical scheme detailed in Section III.A are validated by simulating the experiment of Nogueira et al. (2006a,b) in which the flow field around the nose, film, and wake regions around a Taylor bubble rising in aqueous glycerol in an acrylic column of diameter $32 \mathrm{~mm}$ was measured using particle image velocimetry (PIV) and pulsed shadowgraphy techniques (PST). The fluid has a viscosity of $199 \mathrm{cp}$, density of 1233 $\mathrm{kgm}^{-3}$ and a surface tension of $0.0328 \mathrm{Nm}^{-1}$.

The values of the model dimensionless groups corresponding to these properties are Eo $=189$ and $\mathrm{Nf}=111$. The governing equations were solved using these dimensionless parameters for two aspect ratios, $L=1.32$ and 2. The converged steady shapes, flow patterns, and rise velocities are shown in Figure 3. These results indicate that the rise velocity, film thickness, and flow patterns around a Taylor bubble are independent of volume. The predicted Froude numbers, which is the rise velocity, for the two cases, are 0.3072 and 0.3084 , correspond to deviations of $8.45 \%$ and $8.44 \%$, respectively, from the experiment measured value of 0.3355 . Further comparisons with the experiment were carried out using measurements taken at points around the nose, within the film and below the bottom regions using the results for the aspect ratio $\mathrm{L}=2.0 \mathrm{in}$ a fixed frame of reference.

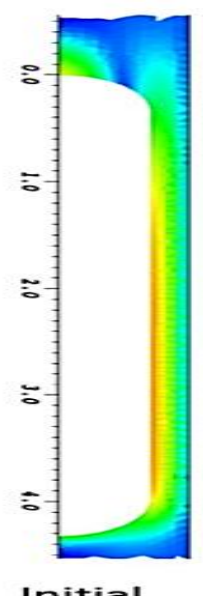

Initial
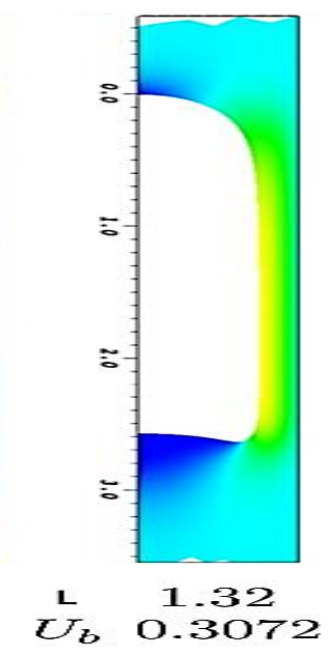

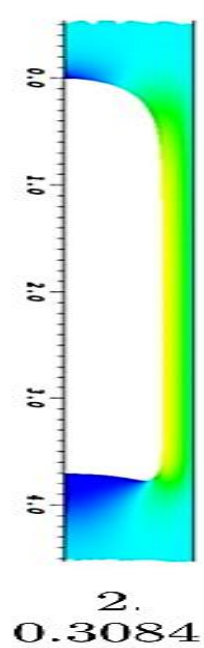

Figure 3: Steady state contour plot around a Taylor bubble rising in liquid of $\mathrm{Nf}=111$ and $\mathrm{Eo}=189$ for aspect $\operatorname{ratios} L=1.32$ and $L=2.00$.

\section{1) Hydrodynamics of nose region}

In the nose region, measurements of axial velocity were taken in the radial direction at three different dimensionless axial distances ahead of the bubble nose, $0.00,0.10$, and 0.20 . Figure 4 shows the experimental velocity profiles for these three locations scaled with the bubble rise velocity and are well predicted by the numerical simulation. The velocity profiles all peak at the origin, affirming that the impact of the rising bubble on the surrounding liquid is most felt at points directly above the nose of the bubble, fixed as the origin, as it pushes through the liquid. This impact decreases farther away from the nose of the bubble as captured by the increase in the flatness of the velocity profile and decrease in the magnitude of the axial velocity as the axial distance from the nose is increased. 


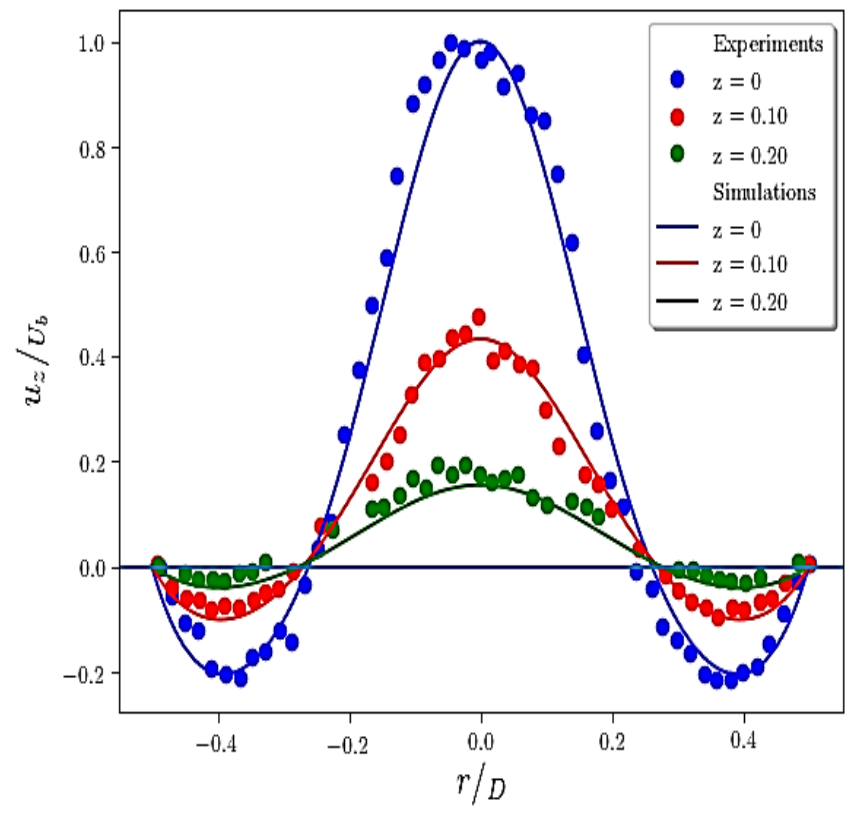

Figure 4: Axial components of velocity as a function of dimensionless radial location ahead of the bubble nose; numerical results (solid lines) compared with experimental PIV measurements (symbols) of Nogueira et al. (2006a, b).

With further increase, a point is reached beyond which the velocity profiles become flat and the magnitude of the velocity is zero. This distance when obtained along the tube axis is known as the stabilisation length ahead of the bubble and it was determined to be 0.4601 corresponding to a deviation of $9.5 \%$ from the experimental value of 0.42 . Figure 5 shows the comparison between the experiment and the numerical results of axial velocity ahead of the bubble nose at fixed radial location. While the numerical results were extracted at the specified radial location, the experiment is the average of the square of the deviation between the local value of the axial velocity and the value for the same radial position far ahead of the bubble obtained at seven different radial positions.

Further comparison in the nose region was carried out by overlaying the plot of the experimental bubble nose shape with the numerical profile of the bubble shape (Figure 6). It is seen that the numerical prediction adequately matches with the experiment.

\section{2) Hydrodynamics of film region}

As Taylor bubble rises in a liquid, it displaces the liquid ahead of it, which flows into the annular space between the bubble and the column wall as a thin film. Starting from the nose of the bubble, which is a stagnation point in a frame of reference that moves with the bubble rise velocity, the radial velocity component increases until it peaks before gradually diminishing, approaching zero, see Figure 7. The region starting from the nose and ending at the point at which the radial velocity on the interface attains its maximum value constitutes the nose of the bubble.
From the point where the radial velocity attains its maximum value to the point where it begins to take a negative value is the film region, which can be divided into the developing film region where the magnitude of the radial velocity is positive and the developed film region where the magnitude is zero.

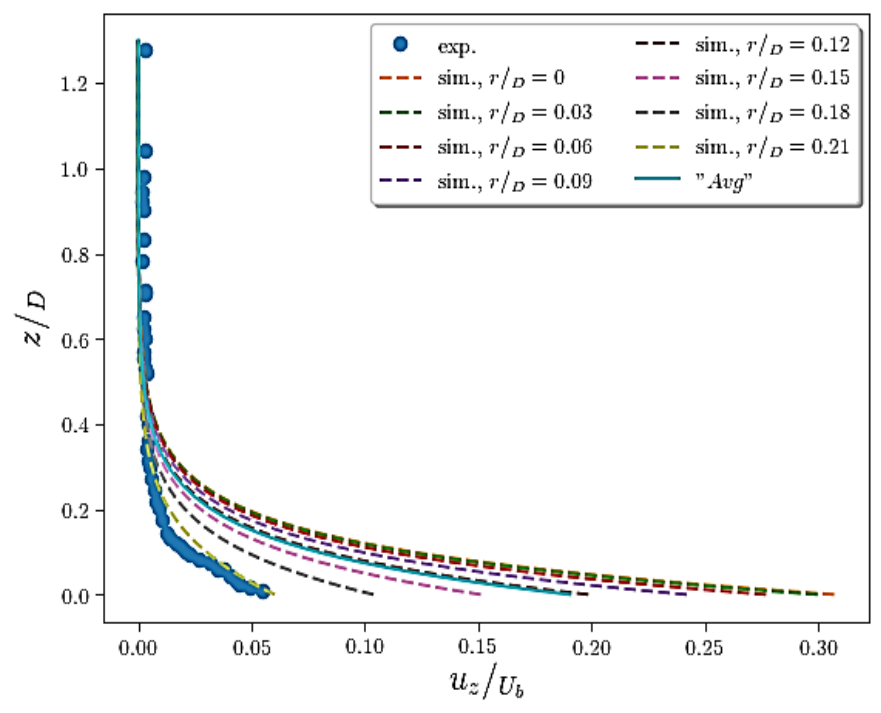

Figure 5: Axial components of velocity as a function of dimensionless axial location ahead of the bubble nose; numerical results (solid lines) compared with experimental PIV measurements (symbols) of Nogueira et al. (2006a,b).

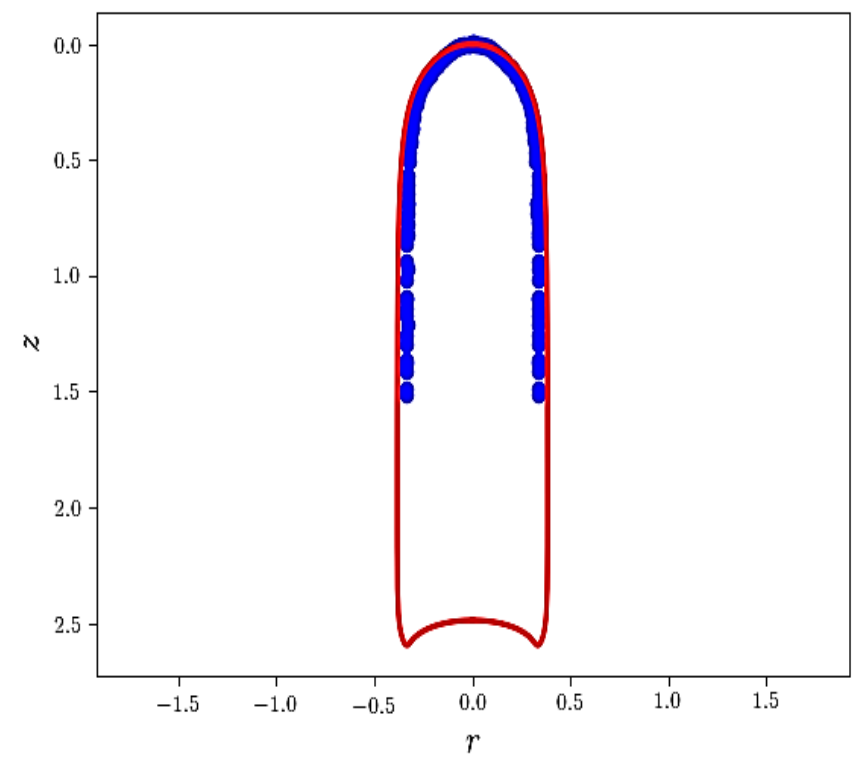

Figure 6: Steady state Taylor bubble shape; numerical results (solid lines) compared with experimental PST measurements (symbols) of Nogueira et al. (2006a, b).

In the fully developed region, the velocity profile and the liquid film thickness are stabilised. The numerical result for the stabilised axial velocity profile is shown in Figure 8, which compares well with the experimental data and the stabilised dimensionless film thickness determined to be 0.1173 that is 
equivalent to a deviation of $2.3 \%$ from the experimental value of 0.12 .

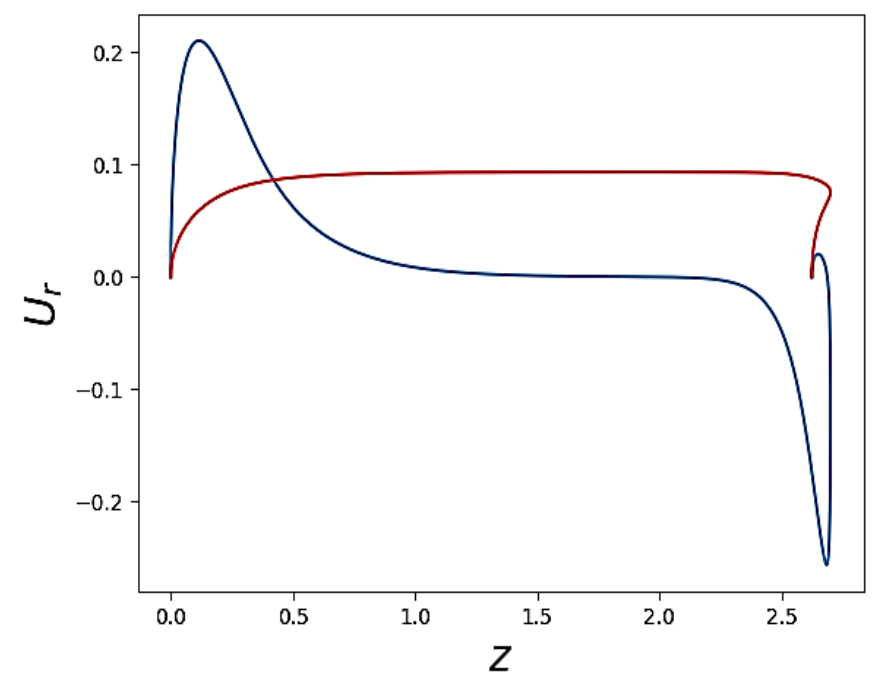

Figure 7: Radial velocity profile (coloured blue) along the interface (coloured red) rotated at angle $-90^{\circ}$.

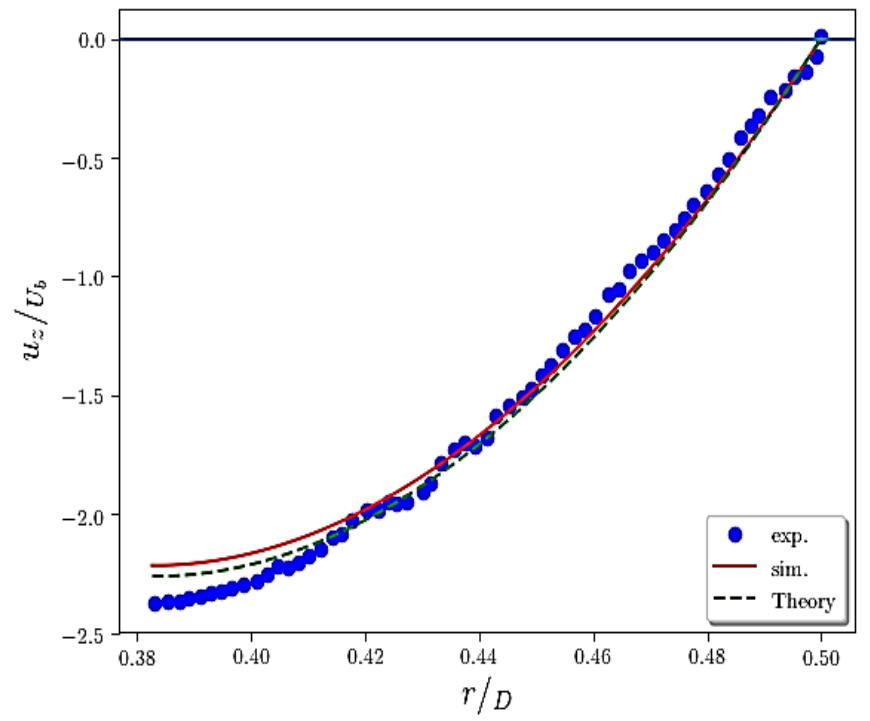

Figure 8: Axial components of velocity in the fully developed film; numerical results (solid lines) compared with theoretical results (broken lines) and experimental PIV measurements (symbols) of Nogueira et al. (2006a, b).

\section{3.) Hydrodynamics of wake region}

As the liquid emerges from the film region into the wake of the bubble, the radial component of its velocity becomes nonzero to redirect the liquid from the film back towards the center of the tube, Figure 7. The axial velocity profiles in the wake of the Taylor bubble at dimensionless axial distances of 0.10 and 0.35 below the bubble bottom are shown in Figure 9.

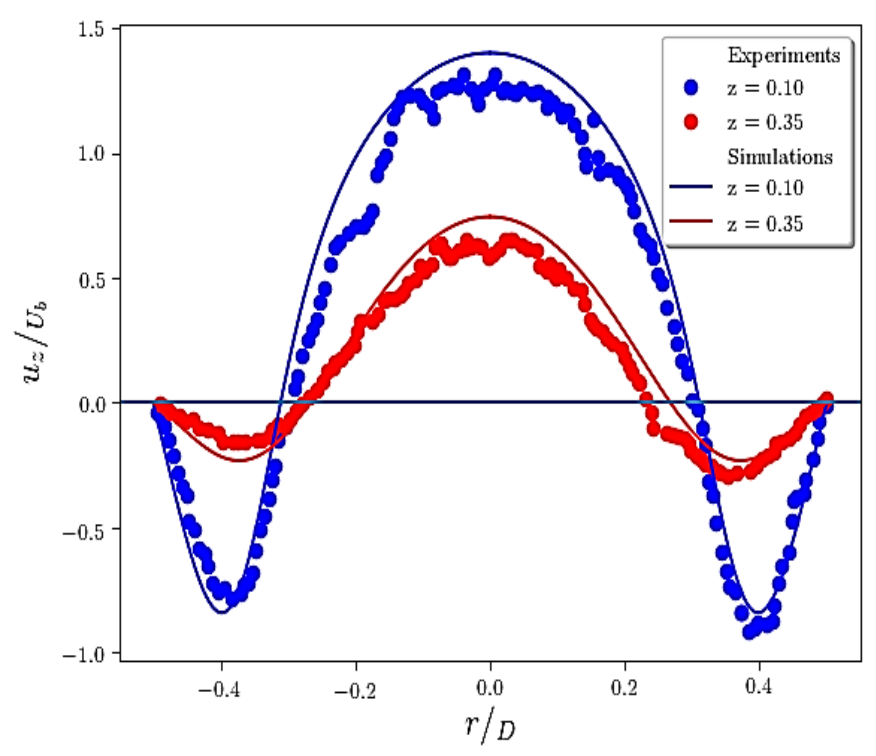

Figure 9: Axial components of velocity at dimensionless distances below the bubble bottom; numerical results (solid lines) compared to experimental PIV measurements (symbols) of Nogueira et al. (2006a, b).

As seen from Figure 9, the predictions from the numerical simulations also reasonably agree well with the experiment. Just as observed for the velocity profiles ahead of the bubble nose, the impact of the wake decreases the farther the distance below the bubble bottom until a point is reached beyond which the velocity profile becomes flat. The minimum distance below the bubble bottom beyond which the impact of the bubble is not felt is the stabilisation length below the bubble bottom and is determined to be 0.345 . The wake flow pattern below the bubble bottom is captured in Figure 10, showing the streamline and vector field around the bubble.

\section{CONCLUSION}

Consistent Petrov-Galerkin finite element scheme for computing steady state liquid velocity and pressure fields, bubble pressure and rise velocity, and interface shape of a rising Taylor bubble in liquid with negligible dynamics in the gas-phase is derived, implemented and validated. The scheme, implemented within FreeFem++ finite element package and which can be adapted to general free surface flow problems, is based on steady state governing equations with implicit treatment of curvature and pseudo-time-step technique for interface deformation. By simulating the experiment of Nogueira et al. (2006a,b) and comparing the results for the velocity profiles at points ahead of the bubble, in the film region and behind the bubble with experiment data, it was shown that the prediction from the scheme is adequate and accurately captures the physics of the problem. Thus, the scheme can be used to further carry out a systematic analysis of the dynamics of a rising Taylor bubble and general free surface flow problems. 


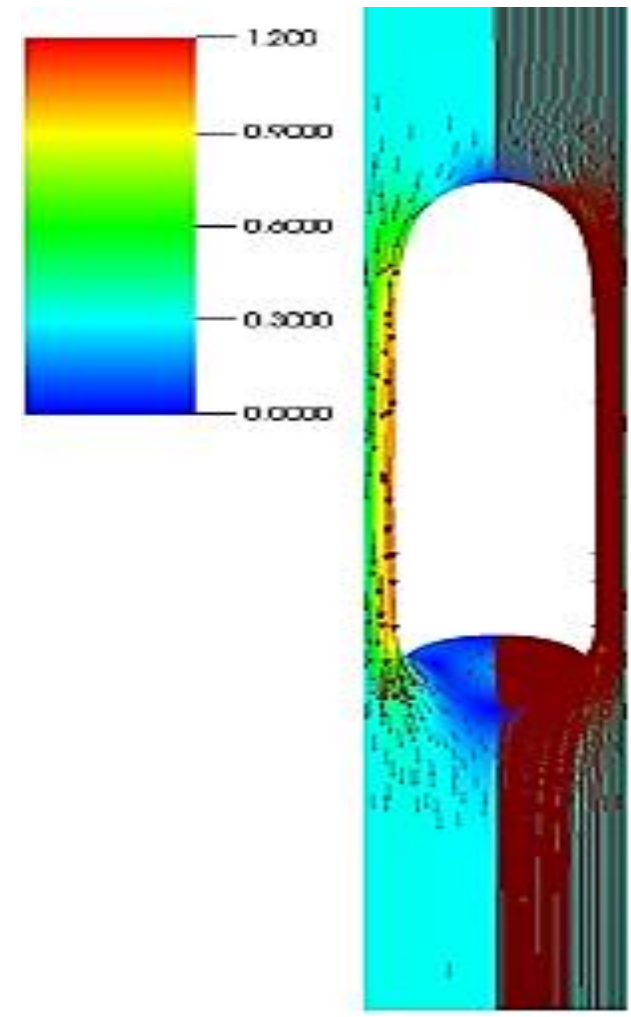

Figure 10: Numerical steady state bubble shape showing the flow vector field (left side) and streamlines (right side) around the bubble.

\section{REFERENCES}

Abubakar, H. A. (2019). Taylor bubble rise in circular tubes: steady states and linear stability analysis. PhD thesis, Imperial College London.

Abubakar, H. A. and Matar, O. K. (2021a). Taylor bubble motion in stagnant and downward-flowing liquids in a vertical pipes.Part I: Steady-states. Submitted to J. Fluid Mech.

Abubakar, H. A. and Matar, O. K. (2021b). Taylor bubble motion in stagnant and downward-flowing liquids in a vertical pipes.Part II: Linear stability analysis. Submitted to J. Fluid Mech.

Anjos, G.; N. Mangiavacchi; N. Borhani and J. R. Thome. (2014). 3D ALE finite-element method for two-phase flows with phase change. Heat Transfer Engineering 35 (5), 537-547.

Ban̈sch, E. (2001). Finite element discretization of the Navier-Stokes equations with a free capillary surface. Numerische Mathematik 88 (2), 203-235.

Bugg, J. D. and Saad, G. A. (2002). The velocity field around a Taylor bubble rising in a stagnant viscous fluid: Numerical and experimental results. Int. J. Multiphase Flow 28, 791-803.

Capponi, A.; M. James and S. Lane. (2016). Gas slug ascent in a strati_ed magma: Implications of flow organisation and instability for Strombolian eruption dynamics. Earth Planet. Sci. Lett., 435:159-170.
Fabre, J. (2016). A long bubble rising in still liquid in a vertical channel: a plane inviscid solution. J. Fluid Mech. 794, R4.

Fazel, M. (2017). CFD simulations of bubble rise in vertical tubes. Msc thesis. Department of Chemical Engineering, Imperial College London.

Feng, J.Q. (2008). Buoyancy-driven motion of a gas bubble through viscous liquid in a round tube. J. Fluid Mech. 609, 377-410.

Feng, J.Q. (2009). A long gas bubble moving in a tube with flowing liquid. Int. J. Multiphase Flow 35, 738-746.

Fraggedakis, D.; M. Pavlidis; Y. Dimakopoulos and J. Tsamopoulos. (2016). On the velocity discontinuity at critical volume of a bubble rising in a viscoelastic fluid. J. Fluid Mech. 789, 310-346.

Funada, T.; D. Joseph; T. Maehara and S. Yamashita. (2005). Ellipsoidal model of the rise of a Taylor bubble in a round tube. Int. J. Multiph. Flow 31, 473-491.

Hecht, F. (2012). New development in FreeFem++. J. Numer. Math. 20 (3-4), 251-265.

Kang, C.W.; S.P. Quan and J. Lou. (2010). Numerical study of a Taylor bubble rising in stagnant liquids. Phys. Rev. E. 81, 1539-3755.

Lizarraga-Garcia, E.; J. Buongiorno; E. Al-Safran and D. Lakehal. (2017). A broadly applicable unified closure relation for Taylor bubble rise velocity in pipes with stagnant liquid. Int. J. Multiph. Flow 89, 345-358.

Llewellin, E.W.; E. Del Bello; J. Taddeucci; P. Scarlato and S.J. Lane. (2012). The thickness of the falling film of liquid around a Taylor bubble. Proc. R. Soc. A 468, 10411064.

Lu, X. and Prosperetti, A. (2009). A numerical study of Taylor bubbles. Ind. Eng. Chem. Res. 48, 242-252.

Magnini, M.; B. Pulvirenti and J. Thome. (2013). Numerical investigation of hydrodynamics and heat transfer of elongated bubbles during flow boiling in a microchannel. Int. J. Heat Mass Trans., 59:451-471.

Nogueira, S.; M.L. Riethmuller; J.B.L.M. Campos and A.M.F.R. Pinto. (2006a). Flow in the nose region and annular film around a Taylor bubble rising through vertical columns of stagnant and flowing Newtonian liquids. Chem. Eng. Sci. 61, 845-857.

Nogueira, S.; M.L. Riethmuller; J.B.L.M. Campos and A.M.F.R. Pinto. (2006b). Flow patterns in the wake of a Taylor bubble rising through vertical columns of stagnant and flowing Newtonian liquids: an experimental study. Chem. Eng. Sci. 61, 7199-7212.

Pepper, D. and Heinrich, J. (2017). The finite element method: basic concepts and applications with MATLAB, MAPLE, and COMSOL. Taylor \& Francis Group.

Pering, T. and McGonigle, A. (2018). Combining spherical-cap and Taylor bubble fluid dynamics with plume measurements to characterize basaltic degassing. Geosciences, 8(2):42.

Pozrikidis, C. (2011). Introduction to theoretical and computational fluid dynamics. Taylor \& Francis Group.

Pringle, C.C.T.; S. Ambrose; B.J. Azzopardi and A.C. Rust. (2015). The existence and behaviour of large diameter Taylor bubbles. Int. J. Multiphase Flow 72, 318-323. 
Reddy, J. and Gartling, D. (2010). The finite element method in heat transfer and fluid dynamics. Taylor \& Francis Group.

Taha, T. and Cui, Z.F. (2006). CFD modelling of slug flow in vertical tubes. Chem. Eng. Sci. 61, 676-687.
Tsamopoulos, J.; Y. Dimakopoulos; N. Chatzidai; G. Karapetsas and M. Pavlidis. (2008). Steady bubble rise and deformation in Newtonian and viscoplastic fluids and conditions of bubble entrapment. J. Fluid Mech. 601, 123-164. 